\title{
Isolated Sacral Vertebral Hydatid Cyst Presenting with Pathological Fracture
}

\author{
Sudipta Mohakud ${ }^{1}$ Biswadeep Ray ${ }^{1} \quad$ Suprava Naik ${ }^{1}$ \\ ${ }^{1}$ Department of Radiodiagnosis, All India Institute of Medical \\ Sciences, Bhubaneswar, Odisha, India
}

\section{J Neurosci Rural Pract 2019;10:565-566}

Hydatid disease is caused by the larval form of Echinococcus granulosus. The reported occurrence of bony hydatidosis is 0.5 to $3 \%$ of all the cases and $50 \%$ of this affects the spine. Spinal echinococcal cyst accounts for $1 \%$ of all cases of hydatid disease. The thoracic spine is most commonly involved followed by the lumbar and then the sacral. ${ }^{1,2} \mathrm{We}$ report a 21-year-old female who presented with nonhealing ulcer in the right leg, gradually progressing back pain radiating to both lower limbs, paraparesis, and urinary complaints, such as urgency and frequency, for a duration of 3 months. She hailed from rural Eastern India and belonged to low socioeconomic strata. She had a history of contact with sheep and cattle. She had no history of any chronic medical illness. There were no hydatid cysts in any other sites in her body, like lung and liver.

Clinical examination revealed local tenderness at the lower lumbosacral region. No soft tissue swelling was appreciated.Neurological evaluation showed lower motor

\author{
Nerbadyswari Deep ${ }^{1}$
}

Address for correspondence Biswadeep Ray, MD, Department of Radiodiagnosis, All India Institute of Medical Sciences, Bhubaneswar 751019, Odisha, India (e-mail: biswadeepray1401@gmail.com).

neuron type paraparesis. There was diminished sensation below $\mathrm{L} 5$ on both sides, and both the lower limbs had power two-fifths. The knee and ankle jerks were absent and plantars were not elicited.

Magnetic resonance imaging (MRI) showed a large expansile lesion of size $7.8 \mathrm{~cm} \times 7.0 \mathrm{~cm} \times 6 \mathrm{~cm}$ with pathologic fracture and collapse of S1 vertebra. The lesion showed centrally clumped $\mathrm{T} 2$ and $\mathrm{T} 1$ hypointense curvilinear membranes with peripherally arranged $\mathrm{T} 2$ hyperintense daugh-

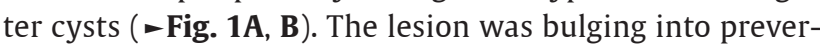
tebral, bilateral paravertebral, epidural (extending from L5 to S2 vertebral levels), and bilateral neural foraminal locations compressing the cauda equina and exiting nerve roots. The posterior surfaces of L5 to S2 vertebrae were scalloped suggesting its long-standing nature. The adjoining intervertebral disc spaces were relatively preserved. These MRI features were characteristic of spinal hydatid disease.
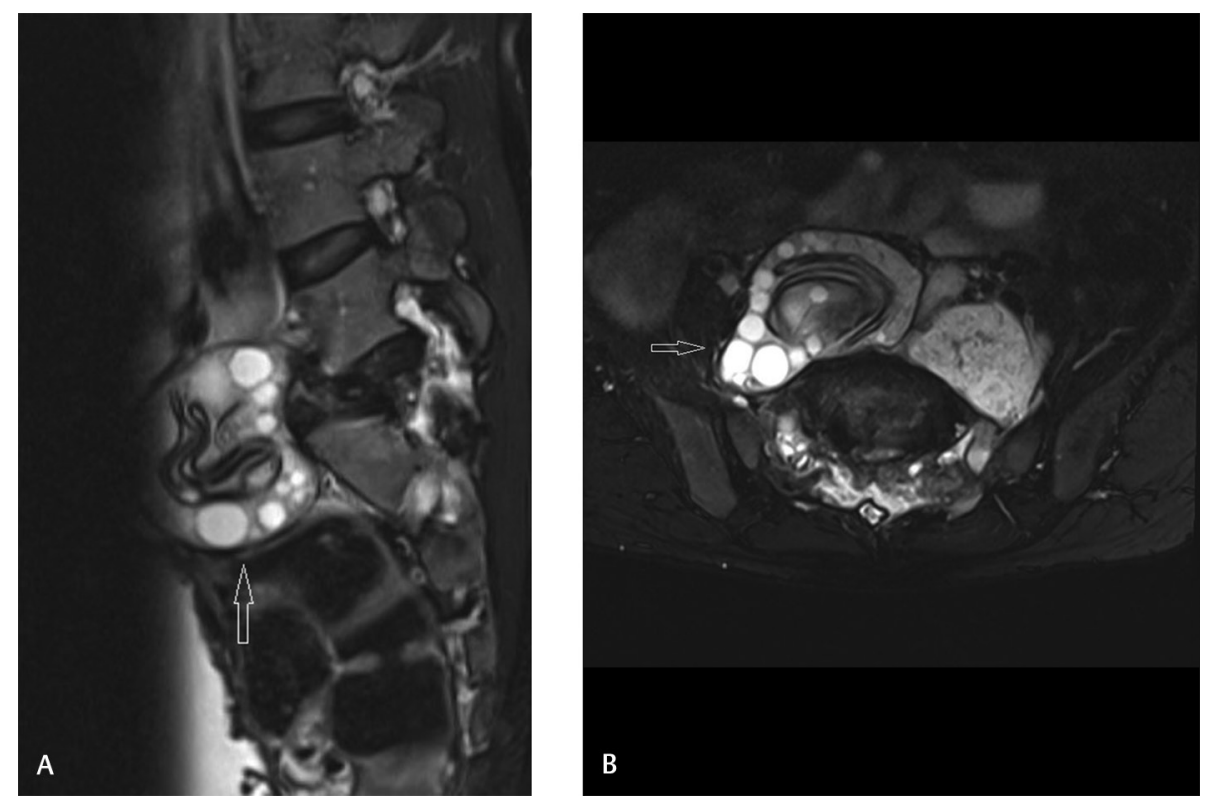

Fig. 1 T2-weighted fat-suppressed sagittal (A) and axial (B) magnetic resonance images show an expansile lesion (arrows) with peripherally arranged hyperintense cysts and centrally clumped curvilinear hypointense membranes, causing collapse of S1 vertebra and extending into the prevertebral and paravertebral regions around L5 to S2 vertebrae. 
The patient underwent L5 to S2 laminectomy with cyst excision and stabilization of S1 fracture. Postoperative histopathologic findings confirmed the diagnosis. Albendazole therapy was initiated, and follow-up at 3 months showed improvement in motor function with regained knee, ankle, and plantar reflexes.

Unusual site of primary hydatid cysts is attributed to the passage of oncospheres through precapillary anastomosis bypassing the lung and liver parenchyma. ${ }^{2}$ The cyst attains huge size due to the absence of pericyst and remains asymptomatic for years. Clinical presentation is nonspecific and occurs due to compression caused by the expanding cyst on the adjacent structures. It may present with local pain, radiculopathy, myelopathy, cauda equina syndrome, and cord compression due to pathological fracture. ${ }^{3}$

The differential diagnoses are primary vertebral tumors, such as aneurysmal bone cyst, arachnoid cyst, epidermoid, schwannoma, metastases, Pott's spine, and vertebral osteomyelitis. MRI is the preferred imaging modality in the diagnosis of spinal hydatid cysts, as it is more accurate in lesion characterization and differentiation from other similar lesions. ${ }^{3}$
This case demonstrates an isolated vertebral hydatid disease with characteristic MRI findings. It can lead to serious complications and should be considered as a possibility in case of a cystic vertebral lesion in an endemic region. Spinal involvement is extremely rare but potentially curable with surgery and antihelminthic drug therapy.

Funding

None.

Conflict of Interest

None declared.

\section{References}

1 Shukla SK, Sharma V, Singh K, Trivedi A. Primary lumbosacral intradural hydatid cyst in a child. J Neurosci Rural Pract 2010;1(2):109-111

2 Alatassi R, Koaban S, Alshayie M, Almogbil I. Solitary hydatid cyst in the forearm: A case report. Int J Surg Case Rep 2018;51:419-424

3 Bhake A, Agrawal A. Hydatid disease of the spine. J Neurosci Rural Pract 2010;1(2):61-62 